\title{
ANNULAR FUNCTIONS IN PROBABILITY
}

\author{
RUSSELL W. HOWELL ${ }^{1}$
}

ABSTRACT. A function $f$ holomorphic in the open unit disk $U$ is said to be strongly annular if there exists a sequence $\left\{C_{n}\right\}$ of concentric circles converging outward to the boundary of $U$ such that the minimum of $|f|$ on $C_{n}$ tends to infinity as $n$ increases. We show here that such functions with Maclaurin coefficients \pm 1 form a residual set in the space of functions with coefficients \pm 1 . We also show that the set of $t$ in $[0,1]$ for which $\sum_{r_{n}}(t) z^{n}$ is strongly annular ( $r_{n}$ is the $n$th Rademacher function) is residual, and measurable with measure either 0 or 1 .

Let $U$ denote the open unit disk, and $H(U)$ the space of holomorphic functions in $U$, where the topology on $H(U)$ is the (complete, metric) topology of almost uniform convergence [4, pp. 142-148]. A function $f$ in $H(U)$ is said to be annular if there exists a sequence $\left\{J_{n}\right\}$ of closed Jordan curves about 0 , converging outward to the boundary $T$ of $U$ such that the minimum of $|f|$ on $J_{n}$ approaches infinity as $n \rightarrow \infty$. If the $J_{n}$ can be taken as circles concentric with $T, f$ is strongly annular. In [5], Kahane shows (using different terminology) that if $\left\{Z_{n}\right\}$ is a complex normal sequence of random variables, and if $a_{n}=n^{\alpha}(\alpha>1 / 2)$, then the function $F_{t}(z)=$ $\sum a_{n} Z_{n}(t) z^{n}$ is a.s. strongly annular. As a consequence, the image of $F$ fills a.s. the whole plane. It is not known if the latter holds for $a_{n}=n^{\alpha}$, $-1 / 2<\alpha \leq 1 / 2$. Since in [2], Bonar and Carroll give a "hard analysis" construction of a strongly annular function whose Maclaurin coefficients are all either $\pm 1,0$, it is natural to let $\alpha=0$ in the above, and to replace $\left\{Z_{n}\right\}$ with the sequence $\left\{r_{n}\right\}$ of Rademacher functions. In this paper we show

Theorem 2. The set $S^{*}=\left\{t \in[0,1]: G_{t}(z)\right.$ is strongly annular $\}$ is re. sidual in $[0,1]$, where $G_{t}(z)=\sum_{\nu=0}^{\infty} r_{\nu}(t) z^{\nu}$, and

Theorem 1. The set $S=\{f \in E: f$ is strongly annular $\}$ is residual in $E$, where $E=\left\{f \in H(U): f(z)=\Sigma a_{n} z^{n}, a_{n}= \pm 1\right\}$.

We remark that although $E$ is not a topological vector space, it is a

Presented to the Society, May 17, 1974 under the title Annular functions and residual sets; received by the editors August $8,1974$. 60F 20.

AMS (MOS) subject classifications (1970). Primary 30-00, 30A10; Secondary

Key words and phrases. Annular function, Rademacher function, residual set, zero-one law.

1 This paper was originally part of the author's doctoral dissertation prepared at The Ohio State University under the direction of Professor Francis W. Carroll. 
complete metric space with the relative topology inherited from $H(U)$. Although Theorem 2 follows from the proof of Theorem 1, we list it separately because its proof is nontrivial, and because it connects with the probabilistic considerations mentioned in the remarks of this paper.

To prove Theorem 1, we use a method of Bonar and Carroll [1]. First, we need

Lemma 1.1。 For every positive real number $R$, there exists a polynomial

$$
P(R ; z)=\sum_{\nu=0}^{N(R)} a(R ; \nu) z^{\nu}
$$

where $a(R ; \nu)= \pm 1, \nu=0,1, \ldots, N(R)$, and $\min _{z \in T}|P(R ; z)|>R$.

Proof. The proof is given by Carroll, Eustice, and Figiel [3]. For completeness, we reproduce their ideas here:

If $P$ is a polynomial of degree $k$ with coefficients \pm 1 , then $Q(z)=$ $P(z) P\left(z^{k+1}\right)$ also has such coefficients. Thus, it suffices to produce a polynomial $P$ with coefficients \pm 1 whose minimum modulus on $T$ is greater than 1. Now $P(z)=z^{4}+z^{3}+z^{2}-z+1$ is such a polynomial, since $\left|P\left(e^{i \theta}\right)\right|^{2}=5+2 \cos 4 \theta+2 \cos 2 \theta$.

Let us proceed to the proof of Theorem 1 . We recall that a basic open neighborhood of a function $f$ in $H(U)$ is given by

$$
V(f, K, \epsilon)=\left\{g \in H(U): \max _{z \in K}|f(z)-g(z)|<\epsilon\right\}
$$

where $K$ is a compact subset of $U$. For $n=2,3, \ldots$ we let

$$
\begin{aligned}
S_{n}=\{f \in E: \text { there exists } r & =r(f) \text { with } 1-1 / n<r<1, \\
& \text { such that }|f(z)|>n \text { for all }|z|=r\} .
\end{aligned}
$$

It is clear that $S=\bigcap_{n=2}^{\infty} S_{n}$; we shall show that each $S_{n}$ is open and dense in $E$.

Suppose that $f$ is in $S_{n}$, so that there exists a real number $r, 1-1 / n$ $<r<1$ such that $|f(z)|>n$ for all $|z|=r$. Taking $K$ to be that circle, and $\epsilon=\min _{z \epsilon K}|f(z)|-n$, we see that all of $V(f, K, \epsilon) \cap E$ lies in $S_{n}$.

Proceeding to the density, we let

$$
f(z)=\sum_{\nu=0}^{\infty} a_{\nu} z^{\nu}, \quad a_{\nu}= \pm 1,
$$

be an arbitrary function in $E$, and we let $V(f, K, \epsilon) \cap E$ be an arbitrary basic open neighborhood of $f$. We may choose $\lambda$ large so that for all $z \in K_{\text {, }}$

$$
\sum_{\nu=\lambda+1}^{\infty}|z|^{\nu}<\epsilon / 2 \text {. }
$$


We let

$$
B=\max _{z \in T}\left|\sum_{\nu=0}^{\lambda} a_{\nu} z^{\nu}\right|
$$

and, using the notation of Lemma 1.1, we set

$$
P\left(2^{\lambda+3}(B+n) ; z\right)=P(z)
$$

where $\operatorname{deg} P(z)=s$. We may select $r, 1-1 / n<r<1$, such that for $|z|=r$,

$$
|P(z)|>2^{\lambda+2}(B+n)
$$

Finally we define $g(z) \in E$ by

$$
g(z)=\sum_{\nu=0}^{\lambda} a_{\nu} z^{\nu}+\left(\frac{P(z)}{1-z^{s+1}}\right) z^{\lambda+1} .
$$

Combining this with (1.1) and (1.2), we see that $g$ lies in $V(f, K, \epsilon) \cap E$. Since $1-1 / n<r<1$, we see by (1.3) and (1.4) that for $|z|=r$,

$$
|g(z)|>\frac{2^{\lambda+2}(B+n)}{2}\left(\frac{1}{2}\right)^{\lambda+1}-B=n,
$$

so that $g$ is also in $S_{n}$.

In what follows, we let $D$ be the dyadic rationals and

$S_{n}^{*}=\{t \in[0,1]:$ there exists $r=r(t)$ with $1-1 / n<r<1$, such that $\left|G_{t}(z)\right|>n$ for all $\left.|z|=r\right\}$,

where $G$ is defined as in Theorem 2 .

Lemma 2。1. If $x \in([0,1]-D) \cap S_{n}^{*}$, there exists $\epsilon>0 \quad(\epsilon=\epsilon(x))$ such that $(x-\epsilon, x+\epsilon) \subseteq S_{n}^{*}$.

Proof. Let $x \in([0,1]-D) \cap S_{n}^{*}$, so that for some $r, 1-1 / n<t<1$, we have that $\left|G_{x}(z)\right|>n$ for all $|z|=r$. Thus, there exists a positive number $k$ and an integer $N$ such that $\left|\Sigma_{\nu=0}^{R} r_{\nu}(x) z^{\nu}\right|>n+k$ for all $R>N,|z|=r$. We may choose $\lambda$ large so that $\lambda>N$, and $\sum_{\nu=\lambda+1}^{\infty}|z|^{\nu}<k$ for all $|z|=r$. Since $x \notin D$, we may choose $\epsilon$ small such that if $|x-t|<\epsilon$, then $r_{\nu}(x)=$ $r_{\nu}(t)$ for $v=0,1, \ldots, \lambda$. For all such $t$, we have for $|z|=r$ that

$$
\left|G_{t}(z)\right|=\left|\sum_{\nu=0}^{\lambda} r_{\nu}(x) z^{\nu}+\sum_{\nu=\lambda+1}^{\infty} r_{\nu}(t) z^{\nu}\right|>n+k-k=n \text {. }
$$

Lemma 2.2. If $x \in[0,1]$, and $\delta>0$ is given, there exists $y \in([0,1]$ D) $\cap S_{n}^{*}$ such that $|x-y|<\delta$. 
Proof. We remark that if $y \in[0,1]-D, y$ may be written uniquely as

$$
y=\sum_{\nu=0}^{\infty} \beta_{\nu} 2^{-(\nu+1)}, \quad \sum_{\nu=0}^{\infty} \beta_{\nu}=\infty, \text { where } \beta_{\nu}=\beta_{\nu}(y)=0 \text { or } 1 .
$$

If $y$ is written this way, then we have that $r_{\nu}(y)=1-2 \beta_{\nu}(y)$.

We recall now that in the proof of Theorem 1 , we were able to construct a function $g(z) \in S_{n}$ whose first $\lambda$ Maclaurin coefficients agreed with an arbitrary function $f(z) \in E . g(z)$ was of the form (1.4), where $P(z)$ was a polynomial of large minimum modulus on $T$. We remark also that since $P(z)$ had coefficients equal to 1 and -1 , the function $g(z)$ had infinitely many coefficients equal to \pm 1 .

Let $x \in[0,1]$, and $\delta>0$ be given. We choose $\lambda$ large such that $\sum_{\nu=\lambda+1}^{\infty} 2^{-(\nu+1)}<\delta$, and consider the function $f(z)=\sum_{\nu=0}^{\infty} r \nu(x) z^{\nu} \in E$. As in Theorem 1, we may construct a function $g(z) \in S_{n}$ such that $g(z)=$ $\sum_{\nu=0}^{\infty} a_{\nu} z^{\nu}$, where, for $\nu=0,1, \ldots, \lambda, a_{\nu}=r_{\nu}(x)$, and infinitely many of the $a_{\nu}$ are equal to \pm 1 . We then define $y \in[0,1]-D$ by

$$
y=\sum_{\nu=0}^{\infty}\left(\frac{1-a_{\nu}}{2}\right) 2^{-(\nu+1)}
$$

Since

$$
x=\sum_{\nu=0}^{\infty}\left(\frac{1-r_{\nu}(x)}{2}\right) 2^{-(\nu+1)}
$$

we have that

$$
|x-y| \leq \sum_{\nu=\lambda+1}^{\infty} 2^{-(\nu+1)}<\delta .
$$

Finally, we see that $y \in S_{n}^{*}$ since $\Sigma_{r_{\nu}}(y) z^{\nu}=g(z)$.

To prove Theorem 2, we note that $S^{*}=\bigcap_{n=2}^{\infty} S_{n}^{*}$, and that by Lemmas 2.1 and 2.2 each $S_{n}^{*}$ contains a dense open subset of $[0,1]$-namely $U\left\{(x-\epsilon, x+\epsilon): x \in([0,1]-D) \cap S_{n}^{*}\right\}$.

Remarks. $S^{*}$ is measurable, since by Lemma 2.1,

$$
S_{n}^{*}=U\left\{(x-\epsilon, x+\epsilon): x \in([0,1]-D) \cap S_{n}^{*}\right\} \cup\left\{x: x \in D \cap S_{n}^{*}\right\} .
$$

Since $S^{*}$ is a tail event, we have by the zero-one law $[5$, p. 6], that its measure is either 0 or 1 . Because of Theorem 2, it would in some sense be "expected" that the measure of $S^{*}$ is 1 . It is not known, however, if this is the case, and since $S_{n}^{*}$ is not a tail event, one may not use the zero-one law to reduce the problem of proving that the measure of $S^{*}$ is 1 to that of merely showing that $S_{n}^{*}$ has positive measure. 


\section{REFERENCES}

1. D. D. Bonar and F. W. Carroll, Annular functions form a residual set, J. Reine Angew. Math. (to appear).

2. - Some examples and counterexamples in annular functions (unpublished manuscript)。

3. F. W. Carroll, D. J. Eustice and T. Figiel, On the minimum modulus of a polynomial (unpublished manuscript).

4. H. Cartan, Elementary theory of analytic functions of one or several complex variables, Editions Scientifiques, Hermann, Paris; Addison-Wesley, Reading, Mass., 1963. MR 27 \# 4911.

5. J.-P. Kahane, Some random series of functions, Heath, Lexington, Mass., 1968. MR 40 \# 8095 .

DEP ARTMENT OF MATHEMATICS, OHIO STATE UINIVERSITY, COLUMBUS, OHIO 43210

Current address: USA Tradoc Systems Analysis Activity, White Sands Missile Range, New Mexico 88002 